\title{
Wearable-object-based Interaction for a Mobile Audio Device
}

\author{
KwanMyung Kim \\ KAIST \\ 335 Gwahangno, Yuseong-gu, \\ Daejeon, 305-701, Korea \\ Kmyung@kaist.ac.kr

\section{Dongwoo Joo} \\ Korea Science Academy of KAIST \\ 899 Dangkam3-dong, Busanjin-ku, \\ Busan, 614-103, Korea \\ lucifer0518@naver.com \\ Kun-Pyo Lee \\ KAIST \\ 335 Gwahangno, Yuseong-gu, \\ Daejeon, 305-701, Korea \\ kplee@kaist.ac.kr
}

\begin{abstract}
In this paper, we explore the possibilities of providing miniaturized audio players with gesture control

capabilities that are based on wearable objects. We selected thirteen wearable objects and used them as interaction surfaces. We used user-centered design methods to collect interaction gestures suited for play, stop, volume up, volume down, previous song, and next song functions. The characteristics and possibility of these interaction gestures are also discussed.
\end{abstract}

\section{Keywords}

Wearable interface, interaction gesture, object-based interaction, surface interaction, user-centered design

\section{ACM Classification Keywords}

H.5.2 User Interfaces: Input devices and strategies, Interaction Style, User-centered design.

\section{General Terms}

Design, Experimentation

\section{Introduction}

As technology has developed, mobile music devices have continued to be miniaturized and nowadays the size of MP3 players can be as small as a fingertip. This trend has led to a challenge in designing intuitive control systems and easy interaction for such devices. Existing devices usually require visual contact to operate the device, utilize 
an additionally attached remote control, or offer buttons that can be sensed through touch. All of these interaction styles however, disturb the user's primary function, such as walking or jogging. In this study we assumed that objects worn in everyday life could act as surrogates to interaction devices. Every wearable object has its own shape and function which implies a mode of interaction for that object. We wanted to investigate what gestures users prefere to control a music device while using wearable objects as a medium. We conducted an exploratory study with users to collect interaction gestures, which is the core difference between our study and other research on wearable interfaces. Many other studies exist aiming to develop wearable interaction devices with interaction styles that were pre-defined by experts (e.g. $[4,8,9]$ ).

In this paper, we present how user defined interaction gestures using wearable objects to control music with an extremely small mobile music player. We also describe the characteristics of the gesture sets for each object that should undergo further study and finally discuss other future work opportunities in this area.

\section{Related Work}

The shift of interfaces from a desktop metaphor to the physical environment has been well documented [3].

Physical surfaces are gradually becoming an interaction media. 'SmartSkin' is an example of interactive surface where all sensing elements can be integrated within the surface [8]. This makes it possible for users to use their bare hands for intuitive interaction. This kind of technology is expected to have a greater impact as normal objects become interaction surfaces and as devices get smaller, because small devices have limited space on which interaction can occur. In the future small device will be as tiny as possible and perhaps even embedded within our bodies or inside an artifact as a small chip [1].

Research on interaction with small devices is related to gestural interaction in mobile and wearable interaction. One method to control a small device is to use the back face as the interface surface [2]. However this requires visual attention for control to proceed. To control mobile music players without looking at them, gesture interfaces on the devices have been developed [6]. The user taps or sweeps to play or sift the volume up or down. Another gestural interface method which functions without dedicated visual attention involves moving a device from a part of the body to another as an interaction method [9]. Although these are all developed for gesture interface for use with mobile devices, they cannot be applied to very small devices, for instance, those which are so small that they are hard to grasp.

For most wearable interfaces, instrumented clothing must be worn. WUW (Wear Ur World) consists of camera and a small projector mounted on a hat and uses hand gestures by tracking the user's hand movement with the camera [5]. Smart clothing supported by small electrical devices and fiber and textile technologies can be worn as normal clothing [7]. And a flexible or a foldable textured pad where patterned vibration generated through writing or contact with a finger has shown a possibility as a new input method for generally wearable items [4].

A similar study to our own, in method at least, has been conducted to show development in surface computing. Interaction gestures were defined by users and evaluated [10]. Conveniently, so far, there have been no studies on defining interaction gestures for wearable objects for use with a very small mobile music player. 


\begin{tabular}{|l|c|l|}
\hline Objects & Type & \multicolumn{1}{|c|}{ Remark } \\
\hline shirt & E.C. & $\begin{array}{l}\text { long sleeve, } \\
\text { no zipper \& button }\end{array}$ \\
\hline pants & E.C. & $\begin{array}{l}\text { Long pants } \\
\text { (users' own) }\end{array}$ \\
\hline footwear & E.C. & $\begin{array}{l}\text { Shoes or sneakers } \\
\text { (users' own) }\end{array}$ \\
\hline hat & G.C. & $\begin{array}{l}\text { visor and a button } \\
\text { on the top }\end{array}$ \\
\hline gloves & G.C. & woolen gloves \\
\hline belt & G.C. & $\begin{array}{l}\text { Leather belt with } \\
\text { simple buckle }\end{array}$ \\
\hline glasses & F.G. & $\begin{array}{l}\text { Steel frame, } \\
\text { plastic legs }\end{array}$ \\
\hline earphone & F.G. & no wire \\
\hline watch & F.G. & $\begin{array}{l}\text { circular panel, } \\
\text { leather belt }\end{array}$ \\
\hline $\begin{array}{l}\text { shoulder } \\
\text { bag }\end{array}$ & F.G. & Middle size \\
\hline necklace & AC. & $\begin{array}{l}\text { Simple, } \\
\text { one pendant }\end{array}$ \\
\hline ring & AC & $\begin{array}{l}\text { Simple, no } \\
\text { ornament }\end{array}$ \\
\hline bracelet & AC & $\begin{array}{l}\text { Simple, no } \\
\text { ornament }\end{array}$ \\
E.C.:Essential Clothing, G.C.: General Clothing, F.G.: \\
Functional Goods, AC.: Accessories
\end{tabular}

table 1. Selected wearable objects

\section{Method}

Our main focus in this research was to explore ways to make simple controls for very tiny audio players. For this end we conducted two studies. The first was to collect interaction gestures on wearable objects by asking users to make gestures for controls. The second was to test interaction gesture sets which user preferred to use. We simulated a walking situation with a tread mill in the experiment room and participants tested gestures they had chosen. An audio feedback to gesture input was set up in a Wizard-of-Oz style interaction, in which the users' actions were interpreted and put into effect by a hidden third party. After the main study we conducted a semi-structured interview with the participants about their experience.

\section{Mobile audio player and its control}

In this study, we pretended that the participants were using a very tiny, invisible mobile audio player. So we simulated an invisible MP3 player with a Wizard-Of-OZ style setup. The common controls of a mobile music player are play, stop, previous song, next song, volume up and volume down, which we tested to elicit interaction gestures from the users.

\section{Wearable objects}

The objects to include in the test were selected by the authors. We collected commonly worn objects and categorized them into four groups; essential clothing, general clothing, functional goods and accessories. We then evaluated them by frequency of use, to select objects for use in the test. We exclude gender specific objects like scarves and earrings. Finally we had selected thirteen objects (table 1). We used the participants' own clothing for two of the essential objects; pants and footwear. All participants wore long pants. Footwear was different among users. Some wore shoes and others wore sneakers however, none wore slippers. Some shirts had zippers and some were shirtsleeves. To make the experiment consistent, we provided long sleeve shirts without any zippers or buttons. Except for the essential clothing, we provided all the other objects. We collected simple and common objects, which could be representatives of those groups of objects. For the earphones, we assumed they would be wireless because they could not be connected to the invisible MP3 player.

Participants

We assumed that most users of MP3 players are teenagers and people in their early 20s'. So Sixteen participants in this age group ( 8 males and 8 females; 5 males and 5 females were high school students and 3 males and 3 females were college students) were recruited. Their average age was 19.6 ( $s d=1.02)$. All of them had experience using MP3 players and were familiar with the typical controls.

\section{Study equipment}

To simulate a walking situation while controlling the invisible MP3 player, we used a treadmill and a Bluetooth Stereo Headset (Samsung SBH700) and a laptop computer connecting to it. A video camera was used for recording the procedure and a beam projector for giving instructions to the participants.

\section{Procedure}

We divided the experiment into two sessions: session 1 collecting gestures, session 2 - testing selected gesture sets in simulated walking. The first session was to collect user-defined interface gestures. Thirteen artifacts were separately shown to participants. And participants were asked to wear them in the way they usually did. In case a participant didn't usually wear or bring a particular artifact, they were asked to simulate the most natural situation they could. We then asked them to show how they would control an MP3 player with the presented 


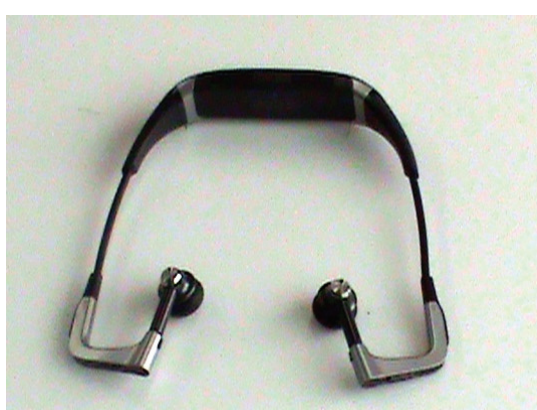

figure 1. Bluetooth headset

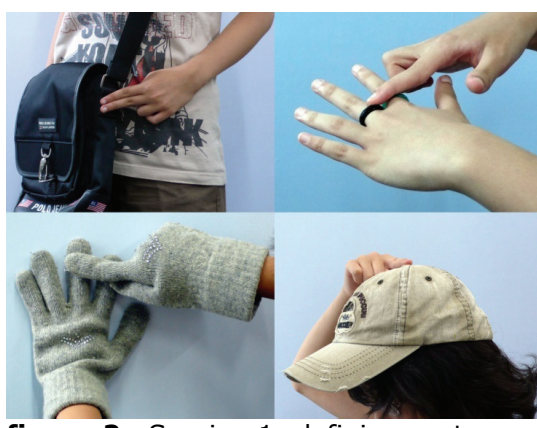

figure 2. Session 1: defining gestures

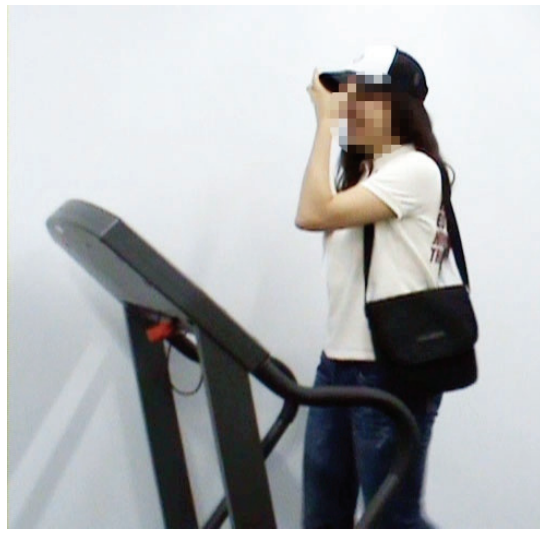

figure 3. Testing gestures on tread mill objects. The given controls were play, stop, previous song, next song, volume up and volume down. When participants presented an input gesture, immediately they were asked to rate it on 7-point Likert scales about the quality and suitability of the gesture with the control.

After completing the first session, the authors sorted objects by rating of the quality and suitability of the gestures for given controls. For each control, objects were ordered from highest to lowest by their ratings. We showed the re-ordered object lists for each control to the participants and then they were asked to select three objects which they preferred to use for the six input controls. The re-ordered objects were shown to them while they made selection. The number of objects to select was more than three, because we didn't want interaction gestures that concentrated on a particular object like a general remote controller. After that, participants were asked to wear the selected objects as they did in normal walking situations. The input gestures for each object they made in the first session were reintroduced to remind them. Then they had a time to warm up to be familiar with the gestures for about three minutes. After that, real time interaction was emulated to test the gestures on the objects. Participants went on the tread mill and set the walking speed at 3 mile per an hour. While they walked, instructions were given on the screen so that the participant made corresponding gestures. The instructions were 'Play', 'Stop', 'Previous title', 'Next title', 'Volume up', and 'Volume down'. Each randomly appeared 5 times. When the participant made a correct gesture, which was the gesture he or she defined, one author controlled a corresponding button on the laptop computer, so that the participant felt that they were getting feedback from the gesture. Finally after finishing this session, we asked each participant about the experience.

\section{Result}

For every object, identical gestures for each control were categorized by reviewing videos. They were grouped into gesture sets for every object. By this we defined gesture sets for every wearable object (Figure 4). Some gestures were really identical among participants. Twelve participants out of sixteen had identical gestures for 'Play/Stop' on the necklace. And nine had identical gestures for 'Previous' and 'Next' on the ring. However gestures for 'Volume up' and 'Volume down' were all different on the gloves.

To decide a representative gesture for each control on each artifact, we listed all gestures by the number of identical appearances and selected the highest valued one as the representative gesture. In the case that multiple gesture sets ranked at the top, we selected all of them as the representatives (e.g. 'Volume up' and 'Volume down' on the belt). And if there was no identical gesture ('Volume up' and 'Volume down' on the gloves) we didn't define a gesture for that control. On the ring and bracelet, gesture sets for different controls overlapped. 'Rotating the ring away from the body' was selected for 'Previous title' and 'Volume up' by different users, as 'Rotate the ring to the body' was for 'Next title' and 'Volume down'. However for the two artifacts, 'Previous title' and 'Next title' had more identical gestures than 'Volume up' and 'Volume down' respectively.

Based on participants' evaluation of the quality and suitability of gestures on objects, user-preferred objects for each gesture control were ranked (Figure 4 6). For each control, the preferred objects were different. As a single combined control the watch was the most preferred. For Play/Stop, the watch, the necklace, the bracelet and the earphones were ranked highly. 


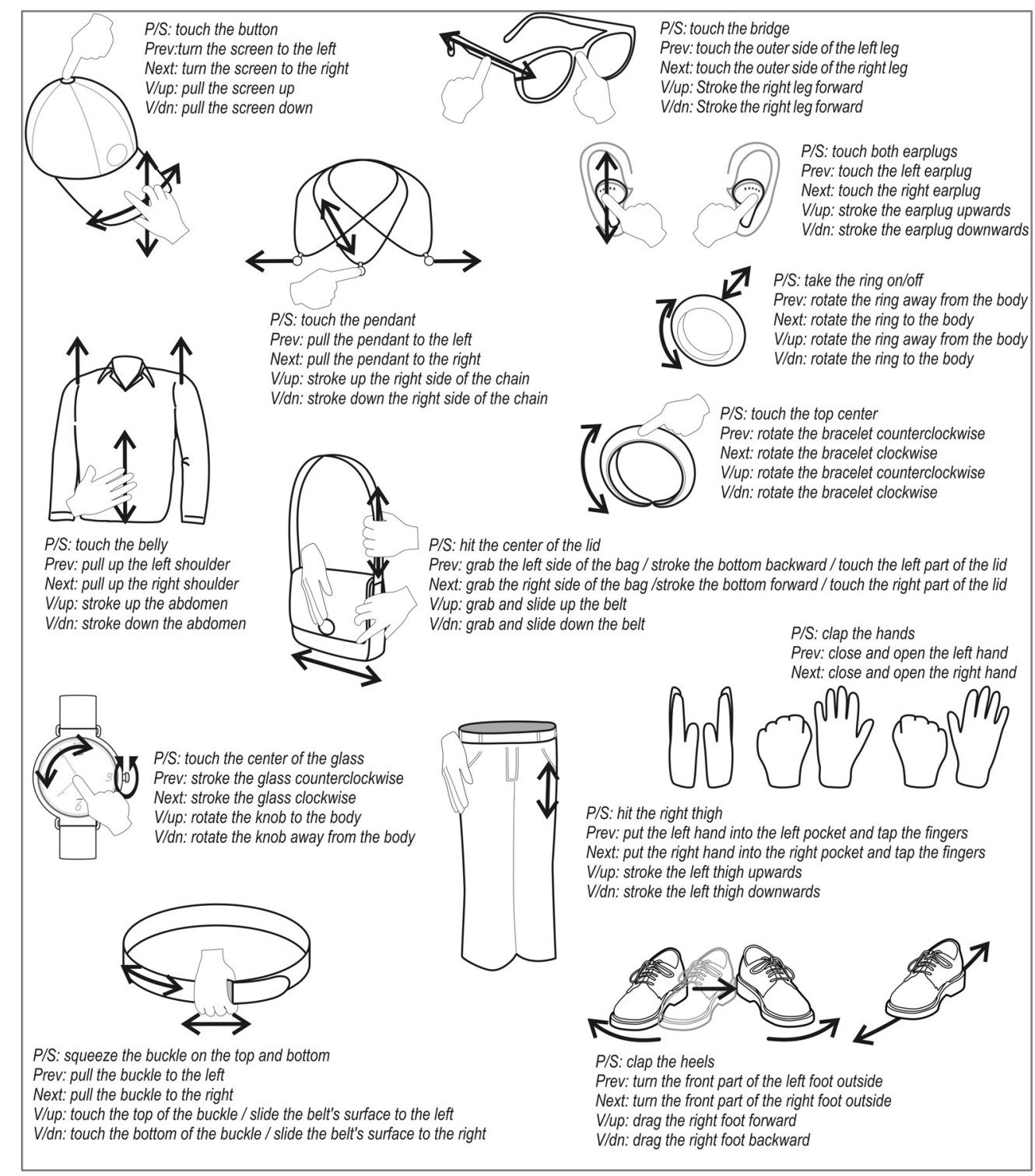

figure 4. Gesture sets for wearable objects (P/S:Play/Stop, Prev:Previous tite, Next:Next title, V/up:Volume up, V/dn:Volume down)
The characteristics of these artifacts were rigid and relatively small. We found that 'Play' and 'Stop' were defined as a same gesture on an object in all cases. They were mostly single tap or touch on a prominent part of objects such as the button on a hat, the pendent of a necklace or the center of the watch. So the representativeness of the results was very high.

All participants selected three objects for session two, which means two paired controls were used on each object; play/stop for one, volume up/volume down for another and previous title/next title for the other.

We found that 'Previous title' and 'Next title's were very much associated with left-right movements. Most participants made gestures related to left movement or left side for 'Previous title' and right movement or right side for 'Next title'. Gestures for 'Volume up' and 'Volume down' had more diverse directional patterns. In different examples, they were related to vertical, left-right,

forward-backward or rotational movements. Interestingly most participants who defined volume up in left and volume down in right or the other way around said that the separation of each direction for volume control was too confusing. After testing the defined gestures in session 2, they wanted to collect both volume up and volume down on one side like on the left side or on the right side. Symmetric placement for 'Previous title/Next title' and 'Volume up/Volume down' caused confusion as well. For example, in case of volume control on the left side of the earphones and navigational control on the right side of the earphones, the participant frequently confused the direction to choose the appropriate gesture. They frequently selected the left side earphone for navigational control or the right side earphone for volume control by mistake. 


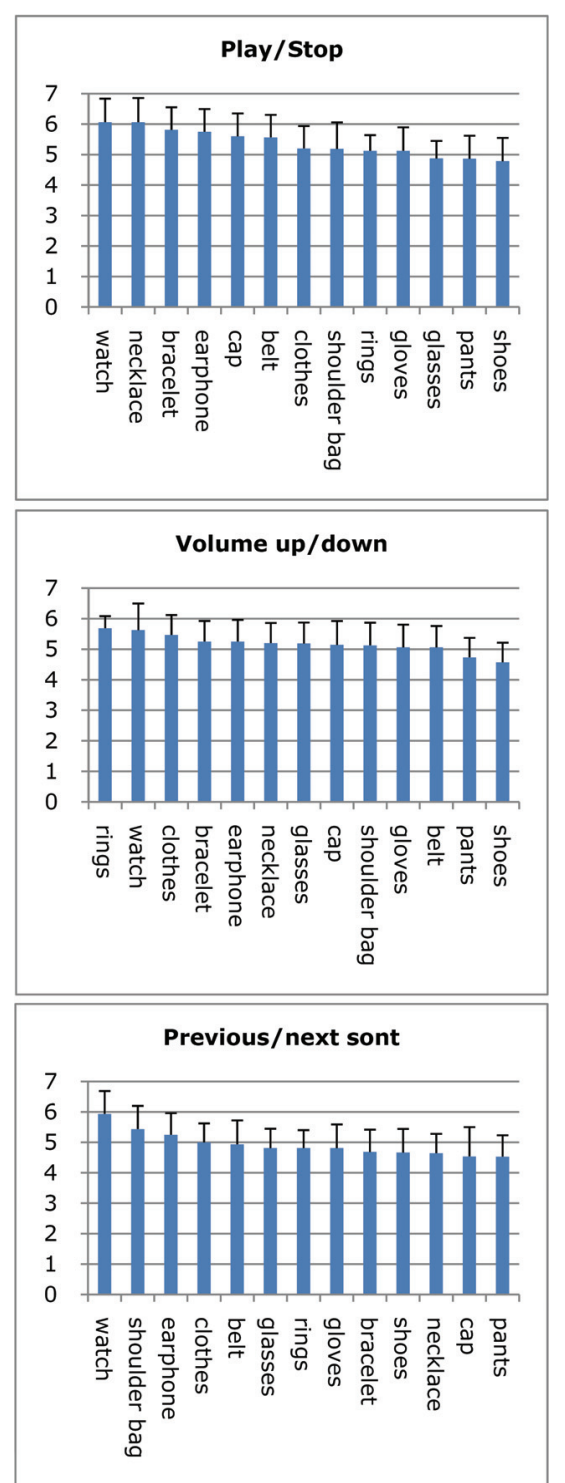

figure 5. Object preference for controls
Most participants mentioned that a wearable-object-based gesture interface was very convenient and natural. They didn't need to pull out the device and the most

advantageous aspect was mentioned as maintaining the main activity flow. They said that they didn't need to slow down their walking and jogging speeds to look at the object for interaction cues. Another advantage is that the interaction gestures are so natural that bystanders are unlikely to be aware when a user is in the process of controlling an audio player. However unintended activating of the controls due to unconscious gestures was an issue that several participants cited as a significant concern. For example a user will touch the center of the watch without any specific purpose, which could cause the music to stop.

\section{Discussion and Future work}

In this study we collected interaction gestures of wearable objects to control music as an initial study to explore the gestural control of miniaturized audio players. We found that there were representative gestures for controls on specific objects, and particular controls matched particular objects very well. Several questions remain; how to verify the representative gestures?, how to refine the gesture sets?, how convenient is it to use gesture interaction and which object is most suitable if only one or two objects are selected? To refine and verify the gestures, the representative gestures should firstly be tested. Some combinations of objects for representative gestures may cause other issues. In this case, rather than an emulated example, working prototypes will work well, because participants will be more elaborately involved in making appropriate gestures with their prototypes. Finally, participants' emotional preference for each gesture according to the objects used should also be studied during the walking situation. By doing so, we can more clearly understand the characteristics of gesturall interaction and the appropriateness of the objects for gesture control.

\section{References}

[1] Auger, J. and Loizeau, J. Audio Tooth Implant. http://www.auger-loizeau.com/index.php?id=7, 2001.

[2] Baudisch, P. and Chu, G. Back-of-device interaction allows creating very small touch devices. In Proc. $\mathrm{CHI}$ 2009, ACM Press (2009), 1923-1932

[3] Ishii, H. and Ullmer, B. Tangible bits: towards seamless interfaces between people, bits and atoms. In Proc. SIGCHI 1997, ACM Press (1997), 234-241

[4] Kim, J.-E., Sunwoo, J., Son, Y.-K., Lee, D.-W. and Cho, I.-Y. A gestural input through finger writing on a textured pad. Ext. Abstracts CHI 2007, ACM Press (2007), 2495-2500

[5] Mistry, P., Maes, P. and Chang, L. WUW - wear Ur world: a wearable gestural interface. Ext. Abstracts CHI 2009, ACM Press (2009), 4111-4116

[6] Pirhonen, A., Brewster, S. and Holguin, C. Gestural and audio metaphors as a means of control for mobile devices. In Proc. SIGCHI 2002, ACM Press (2002), 291298

[7] Rantanen, J., Impio“, J., Karinsalo, T., Malmivaara, M., Reho, A., Tasanen, M. and Vanhala, J. Smart Clothing Prototype for the Arctic Environment. Personal and Ubiquitous Computing, 6, 1 (2002), 3-16.

[8] Rekimoto, J. SmartSkin: an infrastructure for freehand manipulation on interactive surfaces. In Proc. SIGCHI 2002, ACM Press (2002), 113-120

[9] Strachan, S., Murray-Smith, R. and O'Modhrain, S. BodySpace: inferring body pose for natural control of a music player. Ext. Abstracts CHI 2007, ACM Press (2007), 2001-2006

[10] Wobbrock, J. O., Morris, M. R. and Wilson, A. D. User-defined gestures for surface computing. In Proc. CHI 2009, ACM Press (2009), 1083-1092 